\title{
Health worker-only HIV clinic improves care for all
}

I n Malawi, a country with a major shortage of doctors and nurses, improving access to care for HIVpositive health workers is of benefit not only to the workers themselves, but also to their patients. That was one of the messages recently brought to Toronto, Ontario, by Alice Kadzanja in describing a specialized HIV clinic in Malawi.

"Two nurses will have about 100 people in the ward to take care of," says Kadzanja, a nurse at the clinic who is herself HIVpositive. "With HIV, you could be okay today but tomorrow you are sick ... even when you report to duty, your performance is down."

Malawi has one of the lowest densities of health care workers per capita in the world. The country has about two doctors and 38 nurses per 100000 citizens, according to the World Health Organization. Having a clinic that focuses on treating HIV-positive health care workers also improves access to medical services for the general population.

The health worker-focused clinic in Malawi is run by Dignitas International, a Canadian medical humanitarian organization. The aim is to offer a private setting for health care workers away from patients, to reduce wait times and to provide high-quality diagnostic care and treatment. Of the 600 patients, $60 \%$ are health workers. The remaining patients are immediate relatives and other hospital workers, such as cleaners or administrators.

In addition to being exposed to HIV by sexual partners, Malawian health workers contract the virus through needle injuries or blood exposure from cuts, explains Kadzanja. Before HIV education and prophylaxis became available in 2004, health workers who had occupational exposure to HIV and cut themselves "would take no precautions aside from washing and squeezing out the blood," she says. Now, health workers at risk of exposure can take a month-long regimen of prophylaxis and are encouraged to wear masks and gloves.

Kadzanja, who was diagnosed with HIV in 1994, started bringing fellow

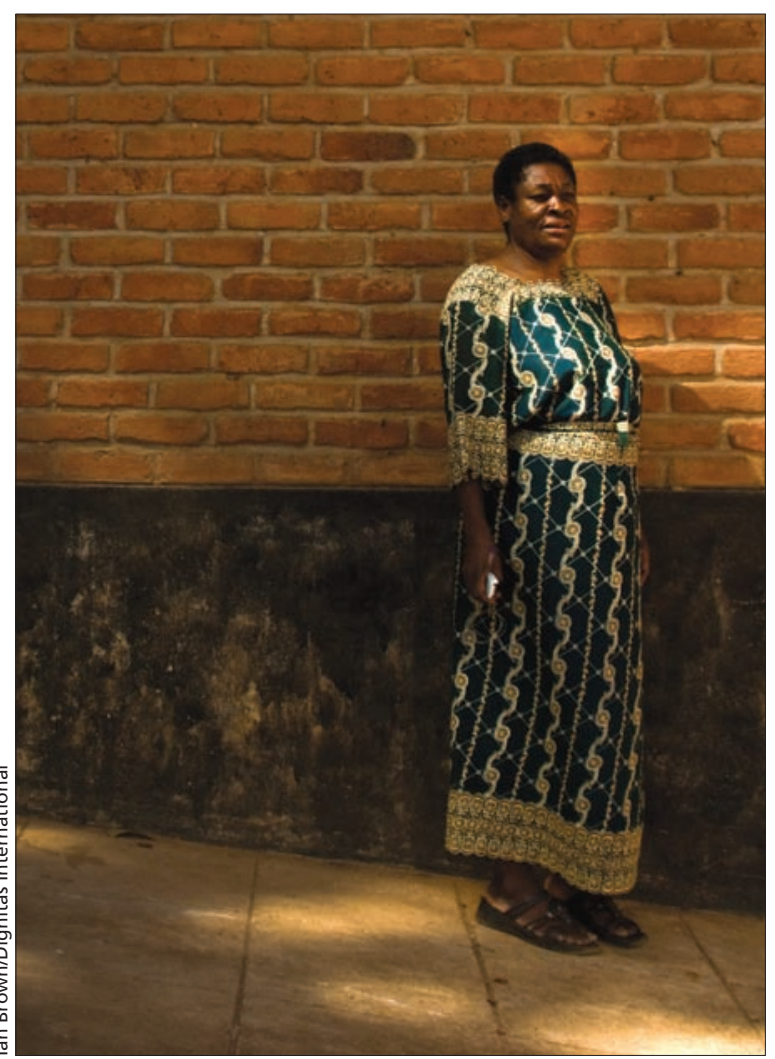

Fear of judgment from patients or fellow health workers deter nurses and doctors with HIV from seeking medical care in general clinics, says Alice Kadzanja, a nurse at a health worker-only clinic in Malawi.

HIV-positive workers to a separate part of the hospital in 2006, creating an "informal separate clinic," according to Dr. Adrienne Chan, a medical advisor with Dignitas.

Fear of judgment from patients or fellow health workers leads nurses and doctors to delay testing or treatment, says Kadzanja. Health care workers also worry that patients will no longer take them seriously. In a survey of health workers in Swaziland, where Africa's first health worker HIV clinic was launched, one nurse said of her patients: "If they know that I am HIV positive, they will be like, "Wow. How can you tell us to [prevent HIV] and you are not doing it?"

After conducting a small study that indicated health workers started treatment much later than the general population, Dignitas formally launched the Health Care Workers clinic at Zomba Central Hospital in 2007. A survey of more than 1300 hospital employees in Zambia also found that health workers sought HIV treatment far away from their places of work, thereby "incurring considerable financial and time costs."

Medical outcomes at the health worker clinic in Malawi "appear better compared to the general HIV clinic," concluded an observational study by Dignitas International and the Malawi Ministry of Health. At the health worker clinic, $4 \%$ of patients on antiretroviral therapy died over a two-year period, compared to $9 \%$ at a general HIV clinic. In addition, $5 \%$ of patients stopped showing up for appointments at the general clinic - possibly because of moving to new clinics, experiencing poor outcomes or unreported deaths - compared to only $1 \%$ at the health worker clinic.

The health worker clinic's success has inspired the opening of two others in the country.

A key difference between the care provided is that the CD4 count of patients at the health worker clinic is checked once or twice a year, whereas patients treated in community clinics and public hospitals tend to be only clinically monitored. Additionally, Dignitas often pays for HIV-related drugs that are costly or out of stock in the public system. - Wendy Glauser, Toronto, Ont.

CMAJ 2014. DOI:10.1503/cmaj.109-4676 